\title{
Modeling Perfusion Dynamics in the Skin During Iontophoresis of Vasoactive Drugs Using Single-Pulse and Multiple-Pulse Protocols
}

Fredrik Iredahl, Veeranjaneyulu Sadda, Liam Ward, Johannes Hackethal, Simon Farnebo, Erik Tesselaar and Folke Sjöberg

\section{Linköping University Post Print}

\section{Tweet}

N.B.: When citing this work, cite the original article.

Original Publication:

Fredrik Iredahl, Veeranjaneyulu Sadda, Liam Ward, Johannes Hackethal, Simon Farnebo, Erik Tesselaar and Folke Sjöberg, Modeling Perfusion Dynamics in the Skin During Iontophoresis of Vasoactive Drugs Using Single-Pulse and Multiple-Pulse Protocols, 2015, Microcirculation, (22), 6, 446-453.

http://dx.doi.org/10.1111/micc.12211

Copyright: Informa Healthcare / Wiley: 12 months http://eu.wiley.com/WileyCDA/

Postprint available at: Linköping University Electronic Press http://urn.kb.se/resolve?urn=urn:nbn:se:liu:diva-121138 
Modeling perfusion dynamics in the skin during iontophoresis of vasoactive drugs using single-pulse and multiple-pulse protocols: the perfusion half-life as an indirect measure of tissue drug metabolism

Fredrik Iredahl ${ }^{1}$, Veeranjaneyulu Sadda ${ }^{1}$, Liam J. Ward ${ }^{1}$, Johannes Hackethal ${ }^{1,2}$, Simon Farnebo ${ }^{1}$, Erik Tesselaar ${ }^{1}$ and Folke Sjöberg ${ }^{1}$

1. Department of Hand and Plastic Surgery, Burns and Intensive care and Department of Clinical and Experimental Medicine, Linköping University, Linköping, Sweden

2. Ludwig Boltzmann Institute for Clinical and Experimental Traumatology, University of Applied Sciences, Vienna, Austria

Running title: Modeling perfusion dynamics in the skin

\section{Corresponding author:}

Fredrik Iredahl, M.D., PhD student

Department of Clinical and Experimental Medicine

Faculty of Health Sciences

Linklty of Health Sc

SE-58185 Linköping

e-mail: fredrik.iredah!@liu.se 


\begin{abstract}
Objective

After iontophoresis of vasoactive drugs into the skin, the decrease in perfusion may relate to drug metabolism. We delivered vasoactive drugs by iontophoresis using different delivery protocols and measured the perfusion half-life using laser Doppler flowmetry (LDF) to study drug metabolism in the skin.

Methods
\end{abstract}

We measured skin perfusion during iontophoresis of $(\mathrm{ACh})$, methacholine $(\mathrm{MCh})$ and noradrenaline (NA) using a single pulse or separate pulses at different skin sites, and during repeated delivery of $\mathrm{ACh}$ at the same site.

Results

Perfusion half-life was 6.1 (5.6-6.6) minutes for ACh and 41 (29-69) minutes for MCh $(\mathrm{p}<0.001)$. The maximum response with multiple pulses of ACh iontophoresis was lower than with a single pulse (30 (22-37) PU vs. 43 (36-50) PU,p<0.001). Vasoconstriction to NA was more rapid with a single pulse than with multiple pulses. The perfusion half-life of ACh decreased with repeated delivery of ACh at the same site (first 16 (14-18), second 5.9 (5.1-6.9), third 3.2 (2.9-3.5) minutes; $\mathrm{p}<0.001)$.

\title{
Conclusions
}

The drug delivery protocol affects cutaneous microvascular responses to iontophoresis, likely as a result of local drug metabolism. Perfusion half-life may be used as an indicator of local drug metabolism in the skin.

Key words: microcirculation, iontophoresis, acetylcholine, vascular pharmacology, skin 


\section{Introduction}

Our current knowledge of microvascular function stems to a large extent from in vitro preparations of isolated vessels and from intravital microscopic studies of whole microvascular beds in animal models. These designs have limitations, which is why accurate, reproducible techniques to assess microvascular function in vivo in humans are sought. In recent years, a number of in vivo techniques have been developed to study microcirculation in the skin. Transdermal iontophoresis is a technique for controlled, non-invasive delivery of drugs to the human skin in vivo $[3,8]$. It is based on the application of a low intensity current, which causes small- and medium-sized molecules of the drug that are contained in an electrode chamber on the skin, to be transported at a higher rate than passive diffusion.

During the past two decades, transdermal iontophoresis of vasoactive drugs has become one of the experimental in vivo human models to study microvascular function in the skin non-invasively under various conditions [19]. Dose-response experiments that are taken from classic in vitro studies can be mimicked by giving the drugs in increased doses, although there are several complicating factors including the complexity of the living tissue, in particular the presence of intact vasculature, blood flow, and circulating vasoactive substances.

In previous studies we have established experimental protocols that resulted in blood flow responses that could be characterized by pharmacodynamic dose-response models for acetylcholine (ACh) and sodium nitroprusside (SNP), two drugs commonly used to test microvascular function. The model makes it possible to derive conventional pharmacodynamic measurements such as $\mathrm{ED}_{50}$, Hill slope, and maximum response from the blood flow responses [19].

One aspect that limits iontophoresis compared with in vitro vessel preparations is that the concentration of the substances given into the tissue cannot easily be measured. Still, knowledge of the inter-relationship between drug kinetics and dynamics is advantageous for various reasons, such 
as for estimating the extent to which drugs are metabolized or transported out of the tissue, for comparing the potency of different drugs delivered by iontophoresis, for a better design of drug delivery protocols, etc. [7]. These considerations have received little attention in most of the studies to date.

We have previously studied the dynamics of perfusion responses and the role of drug removal from the skin during iontophoresis, by using a mechanistic time-response model in which both the kinetics of the drug in the skin and the dynamics of the vascular response to these drugs are taken into account [18]. We found that particularly ACh delivered by iontophoresis is rapidly and continuously removed from the local tissue, but we could not identify the by which mechanism.

In the current study, we hypothesized that the rapid removal of ACh from the local tissue during iontophoresis is mainly by local hydrolysis of the drug by acetylcholinesterase (AChE). Our primary aim, therefore, was to compare the perfusion half-life of ACh (the time needed for the perfusion to decrease by $50 \%$ of the maximum level) with that of methacholine (MCh), a different muscarinic receptor agonist that is more resistant to hydrolysis by AChE [12]. The secondary aim was to study how differences in drug metabolism of ACh and noradrenaline (NA) cause different iontophoresis delivery protocols to result in different microvascular responses. We hypothesized that both ACh and NA would give a stronger response when given with a single pulse, compared with multiple pulses with the same total iontophoretic charge, because of a continuous removal of drugs. Finally, we studied how repeated iontophoresis of ACh at the same skin site affected the microvascular response. We hypothesized that maximal vasodilatation would increase with repeated iontophoresis of ACh (as a result of increased skin permeability) but that the perfusion half-life would remain the same. 


\section{Methods}

\section{Subjects}

Thirty-four healthy, non-smoking volunteers with no previous history of vascular or skin diseases, who were not on any medication (except oral contraceptives), and whose blood pressure was less than $140 / 90 \mathrm{mmHg}$ were recruited. The subjects were asked not to drink anything that contained caffeine or tea, and to refrain from exercise and from using tobacco products on the day of the experiment. All subjects gave their written informed consent to participate. The study was approved by the regional ethics review board at the University Hospital, Linköping, Sweden.

\section{Drugs}

Acetylcholine chloride (Miochol-E, Novartis Healthcare, Denmark), noradrenaline $1 \mathrm{mg} / \mathrm{ml}$ (APL AB, Umeå, Sweden), and sodium chloride $9 \mathrm{mg} / \mathrm{ml}$ (Baxter Medical AB, Kista, Sweden), were obtained from the hospital pharmacy. Methacholine (acetyl- $\beta$-methylcholine powder, purity $>98 \%$ ) was obtained from Sigma Aldrich (St. Louis, MO, USA). Acetylcholine was dissolved in sterile water (experiments 1 and 3) or sodium chloride (experiment 2) to a final concentration of $10 \mathrm{mg} / \mathrm{ml}$. Methacholine was dissolved in sterile water by the hospital's clinical research laboratory to a final concentration $10 \mathrm{mg} / \mathrm{ml}$. All drugs were prepared on the day of the experiments.

\section{Equipment}

Battery-powered iontophoresis controllers (PeriIont, Perimed AB, Järfälla, Sweden) were used to apply a constant electric current through two silver-silver chloride electrodes, which were attached to the skin by double adhesive tape. The drugs were contained in ring-shaped electrodes (PF-383 and LD-611, Perimed AB, Järfälla, Sweden).

A laser Doppler perfusion monitor (PeriFlux 5000, Perimed AB, Järfälla, Sweden) with thermostatic laser Doppler probes (Probe 481-1, Perimed AB, Järfälla, Sweden) was used to 
measure vasodilating responses during iontophoresis of $\mathrm{ACh}$ and $\mathrm{MCh}$. The probes were mounted directly on to the iontophoresis electrodes. The system was calibrated before the study according to the guidelines given by the manufacturer. The probe used in the current study had a fiber separation of $0.25 \mathrm{~mm}$ and collected perfusion data at a depth of about $0.5-1 \mathrm{~mm}$. The bandwidth of the system is $15 \mathrm{kHz}$.

Tissue viability imaging (TiVi, WheelsBridge AB, Linköping, Sweden) was used to measure the vasoconstrictor responses to iontophoresis of NA. The TiVi technique has previously been described in detail [9]. Briefly, the TiVi technique is a camera-based technique that uses polarized light to analyze regions of wavelengths, green (about 500-600 nm) and red (about 600-700 nm), to estimate the concentration of red blood cells in the dermis (an indirect measure of diameter of the vessel). TiVi is more sensitive than laser Doppler flowmetry (LDF) for the measurement of vasoconstriction [14].

\section{Experimental protocols}

The study consisted of three separate experiments, during all of which the subjects lay semisupine, and the volar skin of the forearm was gently cleaned with chlorhexidine ethanol $(5 \mathrm{mg} / \mathrm{ml}$, Fresenius, Uppsala, Sweden). They were then acclimatized for 30 minutes before the measurements began. Iontophoresis electrodes were filled with the solutions of drugs and gently placed on the skin of the volar side of the forearm. When the electrodes were put in place, care was taken to avoid visible veins and irregularities in the skin. Separate sites were used for each delivery of a drug, except in experiment 3, in which ACh was delivered repeatedly to the same site. The room temperature was maintained between 21 and $23^{\circ} \mathrm{C}$ during the experiments.

Experiment 1: Iontophoresis of acetylcholine and methacholine 
Twenty-one subjects (12 of whom were men), mean (SD) age 28 (6) (range 23-40) years and weight 72 (13) (range 52-93) kg, and body mass index (BMI) 23 (3) (range 19-28) participated. Two iontophoresis electrodes (PF-383), one filled with ACh, and another filled with $\mathrm{MCh}$, were placed at separate sites on the volar forearm. Perfusion was measured using LDF. After a baseline period of 5 minutes, $\mathrm{ACh}$ and $\mathrm{MCh}$ were delivered using anodal iontophoresis (0.02 milliamperes (mA) for 10 minutes) using two identical current controllers. At the end of the period of iontophoresis the blood flow was monitored for an additional 10 minutes.

\section{Experiment 2: Iontophoresis of ACh and NA using a single pulse and repeated pulses}

Nine subjects (6 of whom were men) with a mean (SD) age of 24 (3) (range 20-27) years and weight of 64 (9) (range 53-83) kg participated. ACh and NA were delivered by either a single pulse or five separate pulses at four different sites with ACh being delivered on the volar side of one forearm and NA on the other. The order in which they were delivered and which forearm was used were randomized. LDF was used to measure blood flow responses to ACh, while TiVi was used to measure changes in concentration of red blood cells (RBC) caused by NA. After a baseline period of 1 minute, iontophoresis was started. Either a single current pulse of 10 minutes or 5 repeated pulses of 2 minutes, with 1-minute intervals, were used for both drugs. Anodal iontophoresis was used and the current strength was $0.02 \mathrm{~mA}$ throughout the experiment. Vascular responses were measured at baseline, during iontophoresis, and for 10 minutes after the iontophoresis period.

\section{Experiment 3: Repeated iontophoresis of ACh}

Twelve subjects (7 of whom were men) with a mean age of 28 (4) (range 23-36) years and weight of 70 (14) (range 50-89) kg and BMI 22 (3) (range 19-28) participated. One iontophoresis electrode was filled with the ACh solution and placed on the volar side of one of the forearms, avoiding irregularities in the skin and superficial veins. The skin site was then marked with a pen, and after a 10-minute baseline period, ACh was delivered by anodal iontophoresis during a 10-minute period 
using a $0.02 \mathrm{~mA}$ current. Blood flow at the site of delivery was continuously measured with LDF. After iontophoresis, blood flow was measured for an additional 30 minutes, after which a new drugfilled electrode was applied at the same position and the procedure repeated. In total, three 10minute pulses were placed on the same position on the skin, separated by 30 -minute intervals, and new electrodes were used on each occasion.

\section{Statistics}

Responses to ACh and MCh were compared with a two-tailed, paired Student's $t$ tests. In all experiments, except for the one in which noradrenaline was delivered, perfusion responses during the recovery phase were modeled as a function of time using an exponential decay equation with a clearance rate factor $\lambda$. The perfusion half-life, defined as the time for the perfusion to decrease from its initial maximum value to $50 \%$ of its maximum value, was calculated as $\ln (2) / \lambda$. Estimates of perfusion half-life were compared using a sum-of-square F-test.

In experiment 2, a non-linear $\mathrm{E}_{\max }$ model was fitted to the responses to ACh. With the single-pulse protocol, all data points were used for fitting the curve. With the multiple-pulse protocol, only the maximum response after each current pulse was used. Because the decrease in concentrations of RBC during delivery of NA was linear with time, the responses to NA were analyzed by linear regression. Maximum vasoconstriction with the two protocols was compared with a two-tailed, paired Studentr w $t$ test.

Measured perfusion and RBC concentration values are written as mean (SD). Estimated best-fit parameters are given with their $95 \%$ confidence intervals. Data in the figures are presented as mean (SEM). Probabilities of less than 0.05 were accepted as significant. All statistical analyses were made with the aid of GraphPad Prism version 5.02 for Windows (GraphPad Software, San Diego California USA, www.graphpad.com). 


\section{Results}

Experiment 1: Iontophoresis of acetylcholine and methacholine.

The mean $(\mathrm{SD})$ response parameters are presented in Table 1. Baseline perfusion did not differ between ACh and MCh (ACh 2.8 (1.5) PU; MCh 2.8 (1.3) PU, p = 0.95). During iontophoresis, both drugs rapidly increased skin blood flow by similar amounts (ACh 47 (30) PU; MCh 49 (29) PU, $\mathrm{p}=0.98$ ). After iontophoresis of ACh, blood flow rapidly returned towards baseline, whereas after iontophoresis of MCh, recovery was significantly slower (Figure 1). Modeling resulted in an estimated value for the perfusion half-life of $6.1(5.6$ - 6.6) minutes for ACh compared with 41 (29 - 69) min for MCh ( $<$ 0.001). The uncertainty about the perfusion half-life of MCh can be attributed to the slow decay of perfusion during the measurement.

Experiment 2: Iontophoresis of ACh and NA using a single pulse and repeated pulses

\section{Acetylcholine}

The mean (SD) response parameters and best-fit values $(95 \% \mathrm{CI})$ are presented in Table 2. During the baseline period, there was no significant difference in perfusion between the single-pulse and multiple-pulse protocol (10 (4.2) compared with 10 (4.6) PU, p =0.88). After 15 seconds of iontophoresis, perfusion increased gradually. With the single-pulse protocol, perfusion continued to increase until it reached a plateau after 7.5 minutes of iontophoresis (Figure 2). With the multiplepulse protocol the perfusion changed quickly at the beginning and the end of each pulse of current. A maximum response was usually obtained within 30 seconds of the end of a pulse. The best-fit value of the maximum response $\left(\mathrm{E}_{\max }\right)$ with multiple pulses was $30 \%$ lower than the maximum response with the single-pulse protocol (30 (22 - 37) PU compared with 43 (36 - 50) PU, p < 0.001). The perfusion half-life was estimated as $0.60(0.48-0.81)$ after a single iontophoresis pulse, and as $0.90(0.68-1.3)$ after multiple pulses $(\mathrm{p}=0.68)$. 


\section{Noradrenaline}

At baseline, there was no significant difference in the concentration of RBC between the singlepulse and multiple-pulse protocol (88 (70) AU compared with 91 (66) AU, p = 0.50) (Figure 3). During the iontophoresis of NA, there was a significant decrease in the concentration of RBC (linear regression, $\mathrm{p}<0.001$ ) both with the single-pulse and multiple-pulse protocol. The vasoconstriction was significantly more rapid with a single pulse than with multiple pulses (slope $1.1(-1.3--0.91)$ AU/minutes compared with -0.63 (-0.72 - -0.54) AU/minute, $\mathrm{p}<0.001)$. During the 10 minutes post-iontophoresis, concentrations of RBC stabilized, but did not return to baseline. The decrease in concentration of RBC was lower after NA had been delivered with a single pulse (change from baseline -13.9 (9.2) AU), than after multiple pulses (change from baseline -7.8 (8.5) $\mathrm{AU}, \mathrm{p}=0.04)$.

\section{Experiment 3: Repeated iontophoresis of ACh}

The mean (SD) response parameters are presented in Table 3. Mean baseline perfusion was 4.2 (1.2) PU before the start of the first delivery of ACh and increased to 49 (22) PU after the first iontophoresis pulse (Figure 4). During the second iontophoresis pulse, mean perfusion increased more rapidly than during the first pulse, from 18 (20) PU to 68 (43) PU. During the third pulse mean perfusion showed the fastest increase from 6.5 (3.5) PU to 62 (29) PU. The mean baseline perfusion at the beginning of each iontophoresis pulse varied significantly between pulses $(\mathrm{p}=$ 0.045). Although the mean maximum response was lower with the first pulse than with the subsequent pulses, the difference was not statistically significant (one-way ANOVA, $p=0.36$ ).

During the recovery phases, a gradual decrease towards baseline was seen. The estimated perfusion half-life of ACh decreased significantly with each additional pulse of iontophoresis (16 (14 - 18) minutes after the first pulse, $5.9(5.1-6.9)$ minutes after the second pulse, and $3.2(2.9-3.5)$ minutes after the third pulse, $\mathrm{p}<0.001)$. 


\section{Discussion}

Increasing evidence suggests that findings in the microvasculature of the skin reflect those in the systemic microcirculation [10], for example in patients at risk of coronary disease [11] or those with microvascular angina [17]. Iontophoresis is a non-invasive technique for controlled delivery of drugs to the skin [19]. When iontophoresis is used for delivery of vasoactive drugs, microvascular responses in the skin can be measured in parallel using LDF or similar techniques for measurement of optical blood flow. The local concentration of drugs delivered by iontophoresis depends on various factors, which include the rate of delivery, clearance from the skin by the flow of blood, and local metabolism of the drug. Modeling the dynamics of the microvascular responses gives insight in how drug concentrations change in the skin as a result of metabolism or removal from the area of measurement. In the present work we modeled the perfusion response over time during the recovery phase after iontophoresis of different drugs in an initial approach to indirectly study drug metabolism, and to identify the mechanisms by which drugs are eliminated from the skin.

In the first experiment, we compared the perfusion half-life after iontophoresis of $\mathrm{ACh}$ and $\mathrm{MCh}$, which both induce nitric-oxide-dependent vasodilatation by binding to the muscarinic receptor. We found that the perfusion half-life of MCh was longer than that of ACh during the recovery phase, as we had hypothesized. This finding, a more sustained response with MCh, is in line with the results of a previous study in the skin [16], and can be explained by the fact that MCh is more resistant to endogenous AChE, while ACh, on the other hand, is rapidly degraded by $\mathrm{AChE}$ [12]. The significantly longer half-life of MCh also suggests that the clearance of drugs by local blood flow is of less importance during iontophoresis of these muscarinic receptor agonists.

The results of the second experiment highlight why degradation or removal of drugs in the skin is important in iontophoresis studies. In the case of $\mathrm{ACh}$, we found that the microvascular response depends on how the drug is delivered. This is in agreement with the results experiment 1 , which 
indicate that $\mathrm{ACh}$ has a relatively short half-life in the skin. We have previously recommended a continuous, single current pulse for iontophoretic delivery of ACh based on the hypothesis that the short half-life of ACh would mean that it was more effective when delivered with a single pulse than with repeated shorter pulses [19]. The results of this experiment confirmed our hypothesis, as the final blood flow was $30 \%$ lower when ACh was delivered using multiple pulses than with an equal dose (iontophoretic charge) given with a single pulse. Based on these and previous findings, we suggest that iontophoretic delivery of ACh is optimal when a single current pulse is used. We have previously found that a 10 -minute pulse at $0.02 \mathrm{~mA}$ (in total $12 \mathrm{mC}$ ) results in consistent vasodilatory responses while no appreciable non-specific responses arise [4]. The use of a standard delivery protocol for ACh would also facilitate comparison between studies that use iontophoresis of the drug to assess endothelial function.

When NA was given by iontophoresis there was also a stronger vasoconstrictor response with a single-pulse compared to a multiple-pulse drug delivery protocol. There was also more rapid vasoconstriction with a single pulse than with multiple pulses. NA is a catecholamine that, when given transdermally, causes blanching in human skin by constriction of larger vessels in the subpapillary plexus [2]. NA has a half-life of about 1.5 to 3 minutes after intravenous injection [6], and is metabolized through both methylations by catechol-o-methyltransferase and deamination by monoamine oxidase [5]. Given the short half-life, as with $\mathrm{ACh}$, a stronger vasoconstrictive response can indeed be expected after iontophoresis using a single current pulse. Unexpectedly however, vasoconstriction was sustained for at least 10 minutes after the delivery of the drug had stopped. As this is in contrast with the short half-life of NA, it can be speculated that there was a sustained high local concentration of NA in the skin. However it may also be that remaining intracellular mechanisms contributed to the sustained vasoconstriction observed after iontophoresis of NA. 
In the third experiment we studied how repeated iontophoresis of ACh at the same site affected blood flow. We found that the dynamics of the blood flow response during and after iontophoresis of ACh changed with repeated deliveries. With the second and third repetitive deliveries of the drug, a more rapid increase in blood flow was observed after the start of the iontophoresis period, indicating more rapid delivery with each additional pulse. Iontophoresis has been shown to decrease electrical resistance of the skin and to create pores in the skin that facilitate the transport of drugs [1]. It therefore seems likely that the enhanced delivery with subsequent pulses of iontophoresis is related to increased permeability of the skin caused by the electric current of previous pulses.

Unexpectedly, the estimated perfusion half-life decreased significantly with subsequent iontophoretic delivery. The reason for this is not clear. Given the findings of experiment 1 , it is unlikely that the shorter perfusion half-life was caused by increased clearance of drugs by blood flow, although it is possible that the increased permeability of the skin causes a faster removal of the drug from the measurement volume. It could further be speculated that the decrease in perfusion half-life with repeated iontophoresis may have been caused by increase in the local production of AChE, reduced bioavailability of nitric oxide due to flow-induced oxidative stress, or increased production of other vasoactive substances such as endothelin, although we are unaware of any previous studies that have reported such an effect. To further understand this phenomenon we recommend future studies in which specific antagonists are used to study specific pathways involved in the response to ACh.

It was also noted that the perfusion half-life of ACh was much shorter in experiment 2 compared with experiment 1 and 3. The only difference between experiment 2 and the other experiment was the use of sodium chloride as a vehicle for ACh. As sodium chloride, at physiological concentrations, does not have any known effects on the perfusion in the skin, it could be speculated that the presence of sodium and chloride ions in the drug solution during iontophoresis affects the permeability of the skin, thereby changing the response dynamics. 
Again, these findings are relevant for studies in which iontophoresis of ACh is used as a test of endothelial function, as they indicate that responses are affected by repeated iontophoretic delivery of ACh at the same site (for instance before and after a local or systemic intervention), and possibly by the choice of vehicle. Not taking this into account when interpreting perfusion response data may lead to false conclusions.

The current study has a number of limitations: We did not directly inhibit acetylcholine esterase to study the effect on the half-life of ACh in the skin, but instead used two different drugs of which one (ACh) is metabolized and the other (MCh) is not. Possibly, acetylcholine esterase inhibitors could be delivered via iontophoresis as a pretreatment. To further investigate the kinetics of vasoactive drugs in the skin, future studies could include techniques to modify these kinetics, such as tape stripping. Responses could also be analyzed using an alternative skin location, such as the finger pad. Finally, although LFD has an excellent temporal resolution, it has relatively high site-tosite variability and inter-subject variability [13]. To improve the reproducibility of the results, image-based blood flow measuring techniques such as Laser Speckle Contrast Imaging could be used [15].

\section{Conclusion}

We have indirectly studied the metabolism of ACh and NA in the skin by modeling the dynamics of the microvascular responses after delivery of these drugs by iontophoresis, and by comparing the response to $\mathrm{ACh}$ with the response to $\mathrm{MCh}$. The results suggest that $\mathrm{ACh}$ is rapidly metabolized in the skin, so its delivery by iontophoresis, using multiple pulses separated by current-free intervals, results in lower local doses of the drug and weaker responses as a result of its the fast degradation. The delivery protocol was also of importance with iontophoresis of NA, with a single iontophoresis pulse resulting in faster and more vasoconstriction than multiple pulses. Repeated iontophoresis of 
$\mathrm{ACh}$ at the same skin site affected the vascular responses, probably as a result of altered elimination of the drugs. Modeling the vascular responses during iontophoresis therefore makes it possible to gain a better insight into the local kinetics and dynamics of the drug in the skin, which may help with both the design of experimental protocols and the interpretation of results in the assessment of microvascular function.

\section{Perspective}

The choice of drug delivery protocol affects cutaneous microvascular responses to iontophoresis, likely as a result of local drug metabolism. Perfusion half-life may be used as an indicator of local metabolism of drugs in the skin. 
Figure legends

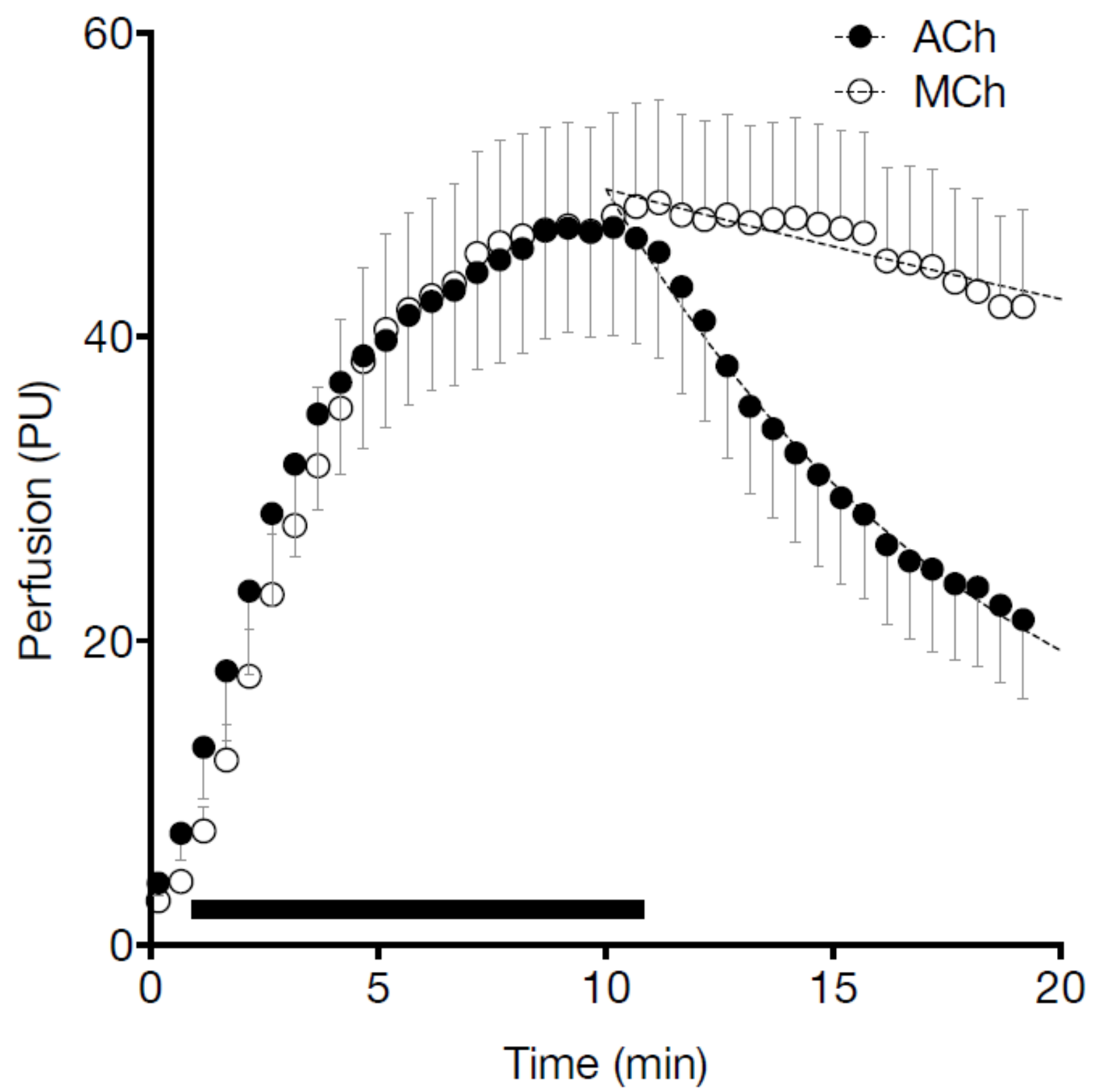

Figure 1. Microvascular responses to acetylcholine ( $\mathrm{ACh}$ ) and methacholine (MCh) given by iontophoresis in the skin and measured with laser Doppler flowmetry (LDF). Bars indicate the period when the current was given. Dashed lines indicate curve-fits obtained using nonlinear modeling of the responses 


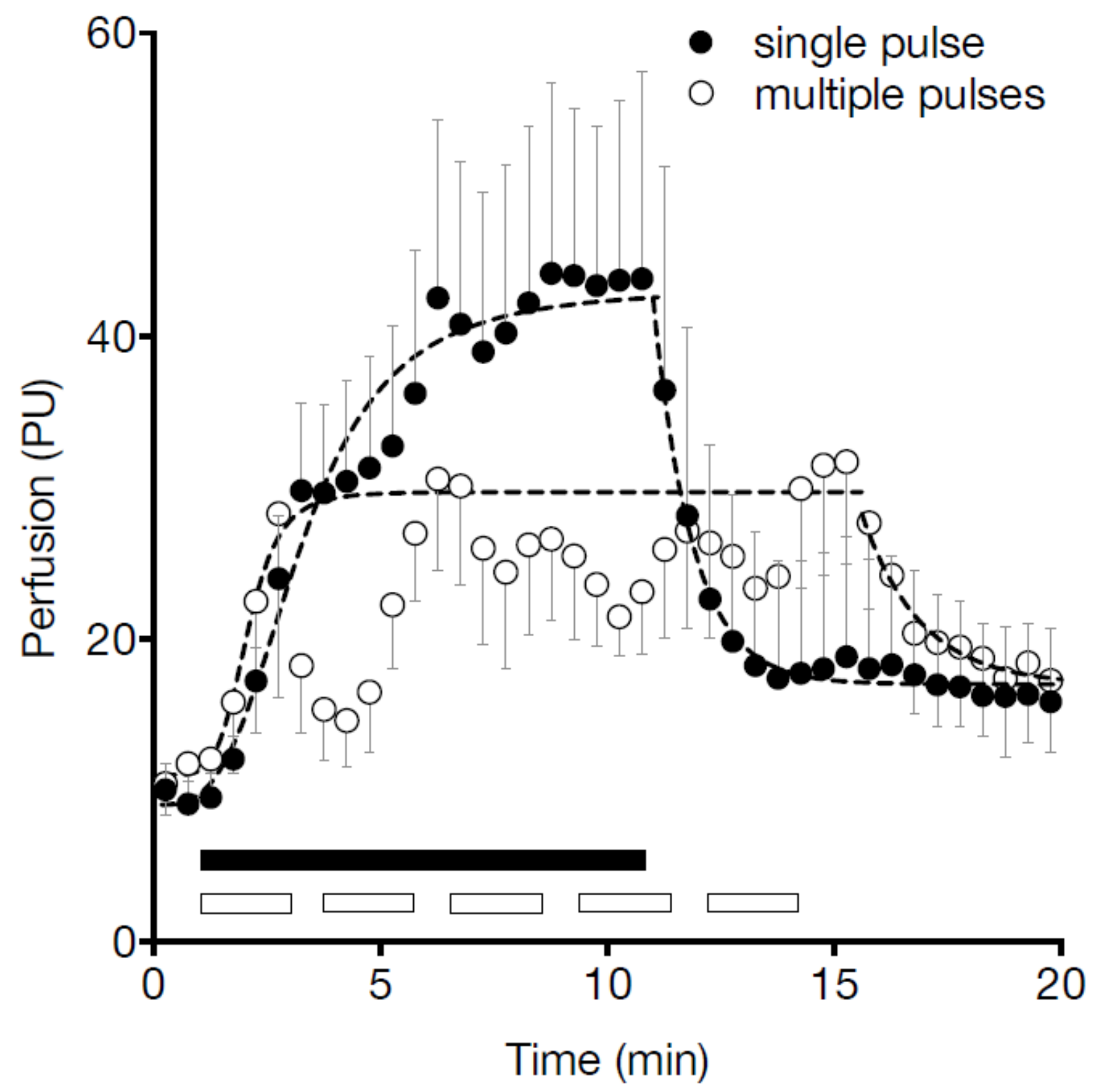

Figure 2. Microvascular responses to iontophoresis of acetylcholine (ACh). The blood flow response to ACh given using a single current pulse (closed circles) was significantly stronger than that with the same dose given as 5 repeated current pulses separated by 1-minute intervals (open circles, $\mathrm{p}<0.001$ ). Bars indicate periods when the current was given during the single-pulse (black) and multiple pulse (white) protocols. Dashed lines indicate curve-fits obtained nonlinear modeling of the responses. 


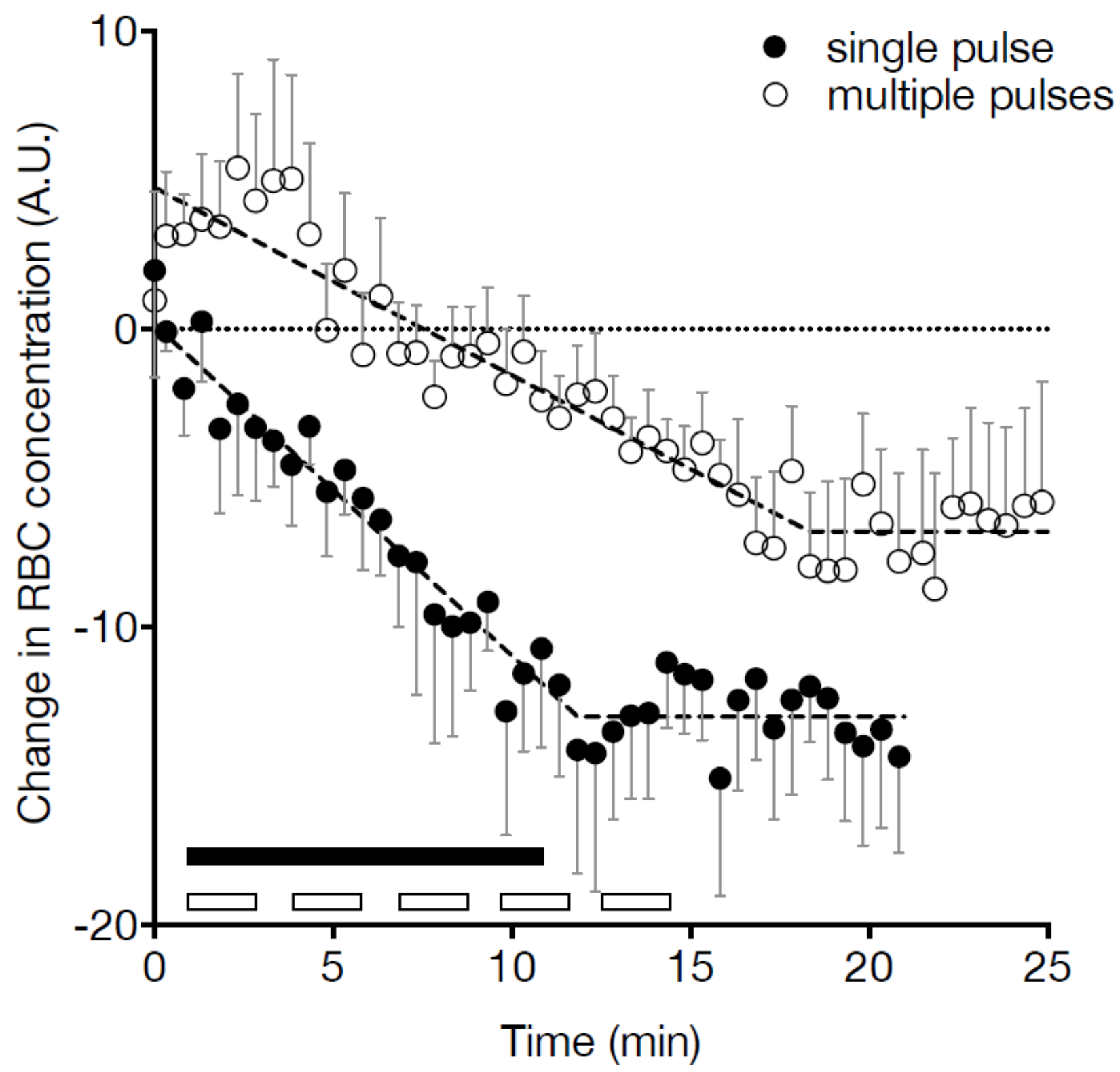

Figure 3. Changes in the concentration of red blood cells in the skin as measured by tissue viability imaging in response to iontophoresis of noradrenaline (NA). The same dose was given using a single current pulse (closed circles) or 5 repeated current pulses separated by 1-minute intervals (open circles). Dashed lines indicate linear regression lines. Bars indicate periods when the current was given during the single-pulse (black) and multiple pulse (white) protocols. 




Figure 4. Microvascular responses to iontophoresis of acetylcholine (ACh) given repeatedly by single 10-minute pulses of iontophoresis. Bars indicate the period when the current was given. Dashed lines indicate curve-fits obtained nonlinear modeling of the responses. 


\section{References}

1. Cullander C. What are the pathways of iontophoretic current flow through mammalian skin? Advanced Drug Delivery Reviews 9: 119-135, 1992.

2. Demis DJ, Zimmer JG, Verhonick PJ, Catalano PM. The pharmacology of human skin. I. Epinephrine and norepinephrine; catecholamine-serotonin combinations. The Journal of investigative dermatology 39: 419-429, 1962.

3. Dixit N, Bali V, Baboota S, Ahuja A, Ali J. Iontophoresis - an approach for controlled drug delivery: a review. Current drug delivery 4: 1-10, 2007.

4. Droog EJ, Henricson J, Nilsson GE, Sjoberg F. A protocol for iontophoresis of acetylcholine and sodium nitroprusside that minimises nonspecific vasodilatory effects. Microvascular research 67: 197-202, 2004.

5. Eisenhofer G, Kopin IJ, Goldstein DS. Catecholamine metabolism: a contemporary view with implications for physiology and medicine. Pharmacological reviews 56: 331-349, 2004.

6. Fitzgerald GA, Hossmann V, Hamilton CA, Reid JL, Davies DS, Dollery CT. Interindividual variation in kinetics of infused epinephrine. Clinical pharmacology and therapeutics 26: 669-675, 1979. 
7. Gabrielsson J, Jusko WJ, Alari L. Modeling of dose-response-time data: four examples of estimating the turnover parameters and generating kinetic functions from response profiles. Biopharmaceutics \& drug disposition 21: 41-52, 2000.

8. Guy RH, Kalia YN, Delgado-Charro MB, Merino V, Lopez A, Marro D. Iontophoresis: electrorepulsion and electroosmosis. Journal of controlled release : official journal of the Controlled Release Society 64: 129-132, 2000.

9. Henricson J, Nilsson A, Tesselaar E, Nilsson G, Sjoberg F. Tissue viability imaging: microvascular response to vasoactive drugs induced by iontophoresis. Microvascular research 78: 199-205, 2009.

10. Holowatz LA, Thompson-Torgerson CS, Kenney WL. The human cutaneous circulation as a model of generalized microvascular function. Journal of applied physiology (Bethesda, Md : 1985) 105: $370-372,2008$.

11. IJzerman R, de Jongh RT, Beijk MA, van Weissenbruch MM, Delemarre-van de Waal HA, Serne EH, Stehouwer CD. Individuals at increased coronary heart disease risk are characterized by an impaired microvascular function in skin. European journal of clinical investigation 33: 536-542, 2003.

12. Kimura K, Low DA, Keller DM, Davis SL, Crandall CG. Cutaneous blood flow and sweat rate responses to exogenous administration of acetylcholine and methacholine. Journal of applied physiology (Bethesda, Md : 1985) 102: 1856-1861, 2007. 
13. Petersen LJ, Zacho HD, Lyngholm AM, Arendt-Nielsen L. Tissue viability imaging for assessment of pharmacologically induced vasodilation and vasoconstriction in human skin. Microvascular research 80: 499-504, 2010.

14. Sauvet F, Mahe G, Chennaoui M, Langrume C, Vasseur M, Abraham P, Leftheriotis G. Acetylcholine chloride as a potential source of variability in the study of cutaneous vascular function in man. Microvascular research 82: 190-197, 2011.

15. Sax FL, Cannon RO, 3rd, Hanson C, Epstein SE. Impaired forearm vasodilator reserve in patients with microvascular angina. Evidence of a generalized disorder of vascular function? The New England journal of medicine 317: 1366-1370, 1987.

16. Tesselaar E, Henricson J, Jonsson S, Sjoberg F. A time-response model for analysis of drug transport and blood flow response during iontophoresis of acetylcholine and sodium nitroprusside. Journal of vascular research 46: 270-277, 2009.

17. Tesselaar E, Sjoberg F. Transdermal iontophoresis as an in-vivo technique for studying microvascular physiology. Microvascular research 81: 88-96, 2011.

18. Wagner JG. Kinetics of pharmacologic response. I. Proposed relationships between response and drug concentration in the intact animal and man. Journal of theoretical biology 20: 173-201, 1968. 
Table 1. Comparison of the characteristics of microvascular responses to iontophoresis of acetylcholine (ACh) and methacholine (MCh). $\mathrm{T}_{1 / 2}$ is the perfusion half-life, defined as the time needed for the perfusion to decrease to $50 \%$ of the maximum response.

\begin{tabular}{lccc} 
& ACh & MCh & p \\
\hline Baseline (PU) & $2.8(1.5)$ & $2.8(1.3)$ & 0.95 \\
Max. response (PU) & $47(30)$ & $49(29)$ & 0.98 \\
$\mathbf{T}_{\mathbf{1} \mathbf{2}(\mathbf{m i n})}$ & $6.1(5.6-6.6)$ & $41(29-69)$ & $<0.001$ \\
\hline
\end{tabular}

Table 2. Comparison of the characteristics of microvascular responses to iontophoresis of acetylcholine (ACh) using a single pulse and multiple pulses of iontophoresis. $\mathrm{E}_{\max }$ is the maximum perfusion response as estimated by the $\mathrm{E}_{\max }$ model.

\section{Single pulse $\quad$ Multiple pulses p}

\section{Acetylcholine}

$\begin{array}{lccc}\text { Baseline (PU) } & 10(4.1) & 10(4.5) & 0.88 \\ \mathbf{E}_{\mathbf{m a x}}(\mathbf{P U}) & 43(36-50) & 30(22-37) & <0.001 \\ \mathbf{T}_{\mathbf{1} / 2}(\mathbf{m i n}) & 0.60(0.48-0.81) & 0.90(0.68-1.3) & 0.68\end{array}$

Noradrenaline

$\begin{array}{lccc}\text { Baseline (AU) } & 88(70) & 91(66) & 0.50 \\ \text { slope (AU/min) } & -1.1(-1.3--0.91) & -0.63(-0.72--0.54) & <0.001 \\ \text { Final change (AU) } & -13.9(9.2) & -7.8(8.5) & 0.04\end{array}$

Table 3. Comparison of the characteristics of microvascular responses to repeated iontophoresis of acetylcholine (ACh) at the same skin site. $\mathrm{T}_{1 / 2}$ is the perfusion halflife, defined as the time needed for the perfusion to decrease to $50 \%$ of the maximum response.

\begin{tabular}{lcccc} 
& $\mathbf{1}^{\text {st }} \mathbf{p u l s e}$ & $\mathbf{2}^{\text {nd }} \mathbf{p u l s e}$ & $\mathbf{3}^{\text {rd }} \mathbf{p u l s e}$ & $\mathbf{p}$ \\
\hline Baseline (PU) & $4.2(1.2)$ & $18(20)$ & $6.5(3.5)$ & 0.045 \\
Max. response (PU) & $49(22)$ & $68(43)$ & $62(29)$ & 0.35 \\
$\mathbf{T}_{1 / 2}(\min )$ & $16(14-18)$ & $5.9(5.1-6.9)$ & $3.2(2.9-3.5)$ & $<0.001$ \\
\hline
\end{tabular}

